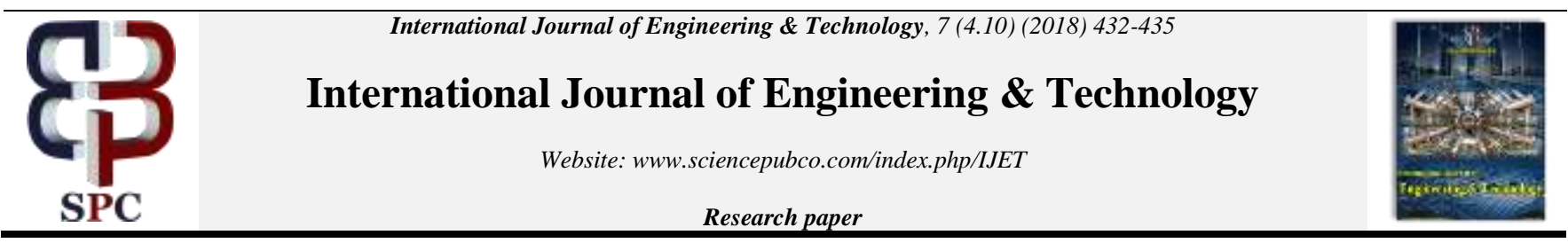

\title{
A Fuzzy Rule based Expert System for T2DM Diagnosis
}

\author{
A.D.Dhivya ${ }^{1}$, A.Felix ${ }^{2 *}$ \\ ${ }^{1}$ Department of Mathematics, Loyola College, Chennai-34 \\ ${ }^{2}$ Mathematics Division, Vellore Institute of Technology, Chennai-600 127 \\ *Corresponding author E-mail: felix.a@vit.ac.in
}

\begin{abstract}
Expert system is an intelligent system to captures the knowledge of a human expert in a specific area. It is capable to make decisions and dealing with ambiguous data. It is used to take an expert view in the absence of a human expertise. Moreover, it is not possible that everyone is expert in every field; to overcome this kind of situation, the expert system is called to handle the complex cases [7]. Due to non availability of the doctor, sometimes patient's life is in risk and lead to death due to not diagnose the disease properly as there are severa diseases whose symptoms are quite similar in initial stage. Hence, the objective of this paper is to design the expert system for diagnosing the diabetes to go for early treatment.
\end{abstract}

Keywords: Linguistic term, Fuzzy number, Fuzzy Expert System, Diabetes.

\section{Introduction}

One of the most significant applications of fuzzy logic is Fuzzy Expert System. Expert System is a branch of applied artificial intelligence, it is used to take an expert view in the absence of a human expertise. Due to the absence of expert in a particular field, FES will solve the real world complex problem. It was first introduced by Kandel [5]. It is an intelligent tool and capable of making decisions and dealing with ambiguous data [10]. FES are applied in many field, medical field is the most important among them. Recently, many researchers designed fuzzy expert system for diagnosing heart disease [1], prostate cancer disease [3]. Webbased fuzzy expert systems are constructed, to deal with human disease diagnosis to overcome various difficulties [2], to predict the risk of diabetic nephropathy [9].

Moreover, soft computing fraternities constructed fuzzy assessment methodology with MATLAB fuzzy logic tool box to diagnose and improve the accuracy of diabetes [4], expert system for all types of diabetes diagnosis based on rule-based [11], casebased and rule-based expert system for type 2 diabetes mellitus management [8]. [7] studied the conception of an efficient fuzzy expert system to minimize the uncertainty effects in different surveillance such as industrial diagnosis, medical diagnosis, video surveillance, economic domain, civil domain, software domain using MATLAB software. Fuzzy expert system and neuro-fuzzy system was derived for gestational diabetes mellitus diagnosis [6]. It is observed that each application has its own inputs and outputs. Thus, the developments of fuzzy expert system is specific for each treated problem. Hence, this paper constructs a new Fuzzy Expert System using Linguistic variable to diagnosis diabetes.

\section{Preliminaries}

Definition 2.1
A fuzzy set $\tilde{A}$ is a subset of a universe of discourse $X$, which is characterized by a membership function $\mu_{\tilde{A}}(x)$ representing a mapping $\mu_{\tilde{A}}: X \rightarrow[0,1]$. The function value of $\mu_{\tilde{A}}(x)$ is called the membership value, which represents the degree of truth that $x$ is an element of fuzzy set $\tilde{A}$.

\section{Definition 2.2}

A fuzzy set $\tilde{A}$ defined on the set of real numbers $\mathrm{R}$ is said to be a fuzzy number and its membership function $\tilde{A}: R \rightarrow[0,1]$ has the following characteristics,

\section{(i) $\tilde{A}$ is convex.}

$$
\mu_{\tilde{A}}\left(\lambda x_{1}+(1-\lambda) x_{2}\right) \geq \min \left(\mu_{\tilde{A}}\left(x_{1}\right), \mu_{\tilde{A}}\left(x_{2}\right)\right), \forall x \in\left[x_{1}, x_{2}\right], \lambda \in[0,1] .
$$

(ii) $\tilde{A}$ is normal if $\max \mu_{\tilde{A}}(x)=1$.

(iii) $\tilde{A}$ is piecewise continuous.

\section{Definition 2.3}

The $\alpha$-cut of the fuzzy set $\tilde{A}$ of the universe of discourse $X$ is defined as $\tilde{A}_{\alpha}=\left\{x \in X / \mu_{\tilde{A}}(x) \geq \alpha\right\}$, where $\alpha \in[0,1]$.

\section{Definition 2.4}

A triangular fuzzy number $\tilde{N}$ can be defined as a triplet $(1, \mathrm{~m}, \mathrm{r})$ and the membership function $\mu_{\tilde{N}}(x)$ is defined as:

$\mu_{\tilde{N}}(x)=\left\{\begin{array}{cc}0 & x<l \\ \left(\frac{x-l}{m-l}\right) & l \leq x \leq m \\ \left(\frac{r-x}{r-m}\right) & m \leq x \leq r \\ 0 & x>r\end{array}\right.$

Where $l, m, r$ are real numbers and $l \leq m \leq r$. 


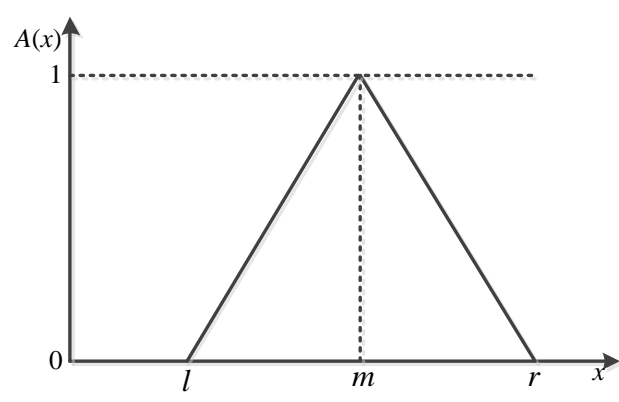

Fig.1: Triangular Fuzzy Number

\section{Definition 2.5}

A trapezoidal fuzzy number $\tilde{A}$ can be defined as $\left(a_{1}, a_{2,}, a_{3}, a_{4}\right)$ and the membership function is defined as

$\mu_{\hat{A}}(x)=\left\{\begin{array}{cc}\left(\frac{x-a_{1}}{a_{2}-a_{1}}\right) & a_{1} \leq x \leq a_{2} \\ 1 & a_{2} \leq x \leq a_{3} \\ \left(\frac{a_{4}-x}{a_{4}-a_{3}}\right) & a_{3} \leq x \leq a_{4} \\ 0 & a_{1} \leq 0 \& a_{4} \geq 0\end{array}\right.$

Where $a_{1}, a_{2}, a_{3}, a_{4}$ are real numbers $a_{1} \leq a_{2} \leq a_{3} \leq a_{4}$.

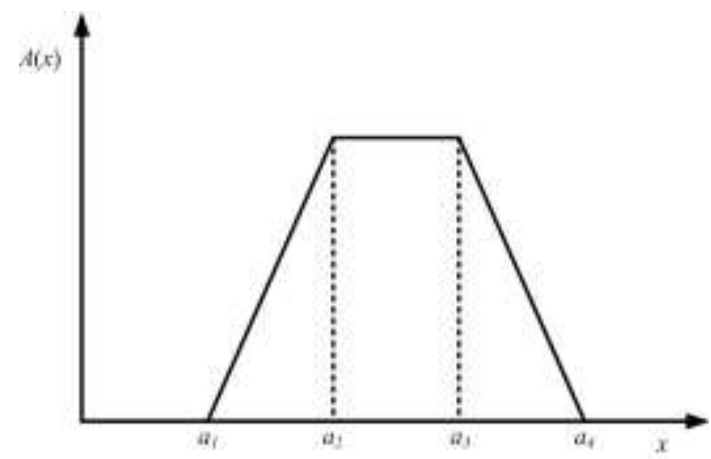

Fig. 2: Trapezoidal Fuzzy Number

\section{Definition 2.6}

Linguistic Variables and Hedges: [12,13] Linguistic variables are the root of fuzzy set theory. A linguistic variable is called fuzzy variable. The statement "Low Density Lipoprotein" is very high implies that the linguistic variable "Low Density Lipoprotein" takes the linguistic value "very high". In fuzzy expert systems, linguistic variables are used in fuzzy rules.

\section{Methodology}

This Fuzzy Expert System was designed in MATLAB tool with the help of Dr.Sebastina (MBBS) from Sri Manakula Vinayagar Medical College (SMVNC), Pondicherry Kalitheerthalkuppam, Madagadipet, Pondicherry. This system has 1200 rule base, the linguistic term of five input variables and one output variable are represented by trapezoidal and triangular fuzzy number respectively.

\subsection{Input Variables}

In this system, we have five input variables such as AGE, HDL, LDL, BW, PA, are given by Trapezoidal Fuzzy Number using classified linguistic variables.

IV -Age: $^{-}$
It is classified into five linguistic variables are described as follows. Consider

$$
\begin{aligned}
& \mu_{\text {Adol }}(x)=\left\{\begin{array}{cc}
1 & x \leq 15 \\
\frac{20-x}{5} & 15 \leq x \leq 20 \\
0 & x \geq 20
\end{array} \quad \mu_{\text {Youmg }}(x)=\left\{\begin{array}{cc}
\frac{x-15}{5} & 15 \leq x \leq 20 \\
1 & 20 \leq x \leq 30 \\
\frac{35-x}{5} & 30 \leq x \leq 35 \\
0 & x \leq 15, x \geq 35
\end{array}\right.\right. \\
& \mu_{\text {Middle }}(x)=\left\{\begin{array}{cc}
\frac{x-30}{5} & 30 \leq x \leq 35 \\
1 & 35 \leq x \leq 45 \\
\frac{50-x}{5} & 45 \leq x \leq 50 \\
0 & x \leq 35, x \geq 50
\end{array} \quad \mu_{\text {Old }}(x)=\left\{\begin{array}{cc}
\frac{x-45}{5} & 45 \leq x \leq 50 \\
1 & 50 \leq x \leq 60 \\
\frac{65-x}{5} & 60 \leq x \leq 65 \\
0 & x \leq 45, x \geq 65
\end{array}\right.\right. \\
& \mu_{\text {Vold }}(x)=\left\{\begin{array}{cc}
0 & x \leq 60 \\
\frac{x-60}{5} & 60 \leq x \leq 65 \\
1 & x \geq 65
\end{array}\right.
\end{aligned}
$$

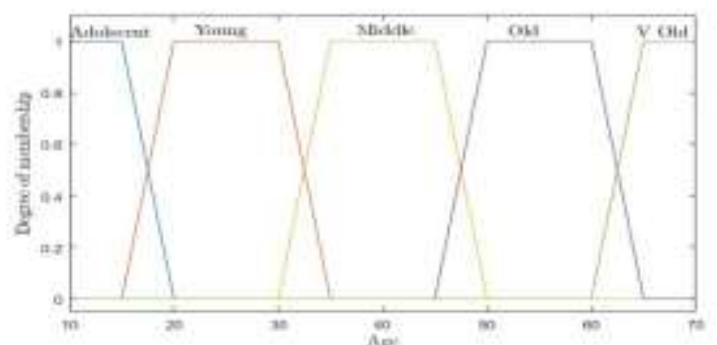

Fig. 3: Classification of Age

Lipoproteins:

The two types of lipoproteins are HDL (Good Cholesterol) and LDL (Bad Cholesterol).

\section{IV $_{2}$-High Density Lipoprotein:}

HDL protects against heart disease by taking bad cholesterol out of our blood and keeping it from our building up in our arteries.

$$
\begin{aligned}
& \mu_{\text {Low }}(x)=\left\{\begin{array}{cc}
1 & x \leq 35 \\
\frac{40-x}{5} & 35 \leq x \leq 40 \\
0 & x \geq 40
\end{array}, \quad \mu_{\text {Medium }}(x)=\left\{\begin{array}{cc}
\frac{x-35}{5} & 35 \leq x \leq 40 \\
1 & 40 \leq x \leq 50 \\
\frac{55-x}{5} & 50 \leq x \leq 55 \\
0 & x \leq 35, x \geq 55
\end{array}\right.\right. \\
& \mu_{\text {High }}(x)=\left\{\begin{array}{cc}
0 & x \leq 50 \\
\frac{x-50}{5} & 50 \leq x \leq 55 \\
1 & x \geq 55
\end{array}\right.
\end{aligned}
$$

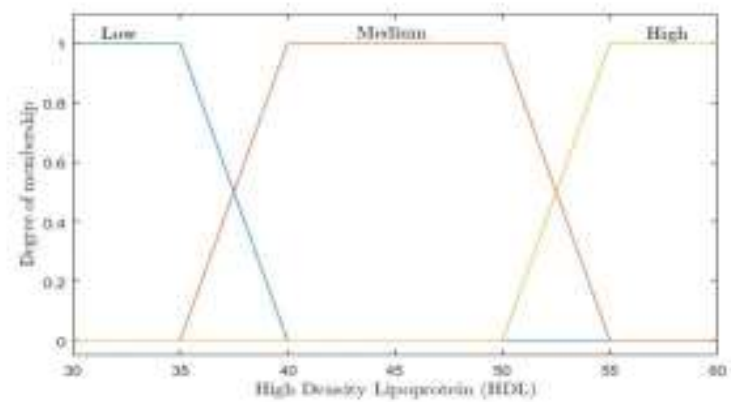

Fig. 4: Classification of HDL 


\section{IV $_{3}$-Low Density Lipoprotein:}

It can build up on the walls of our arteries and increase our chances of getting heart disease. If our LDL is 175 or more, it is considered very high.

$$
\begin{aligned}
& \mu_{\text {Optimal }}(x)=\left\{\begin{array}{cc}
1 & x \leq 95 \\
\frac{100-x}{5} & 95 \leq x \leq 100 \\
0 & x \geq 100
\end{array} \quad, \quad \mu_{\text {Bind }}(x)=\left\{\begin{array}{cc}
\frac{x-95}{5} & 95 \leq x \leq 100 \\
1 & 100 \leq x \leq 120 \\
\frac{125-x}{5} & 120 \leq x \leq 125 \\
0 & x \leq 95, x \geq 125
\end{array}\right.\right. \\
& \mu_{\text {BlindHigh }}(x)=\left\{\begin{array}{cc}
\frac{x-120}{10} & 120 \leq x \leq 130 \\
1 & 130 \leq x \leq 140 \\
\frac{150-x}{10} & 140 \leq x \leq 150 \\
0 & x \leq 120, x \geq 150
\end{array} \quad \mu_{\text {High }}(x)=\left\{\begin{array}{cc}
\frac{x-145}{10} & 145 \leq x \leq 155 \\
1 & 155 \leq x \leq 165 \\
\frac{175-x}{10} & 165 \leq x \leq 175 \\
0 & x \leq 145, x \geq 175
\end{array}\right.\right. \\
& \mu_{\text {VHigh }}(x)=\left\{\begin{array}{cc}
0 & x \leq 170 \\
\frac{x-170}{5} & 170 \leq x \leq 175 \\
1 & x \geq 175
\end{array}\right.
\end{aligned}
$$

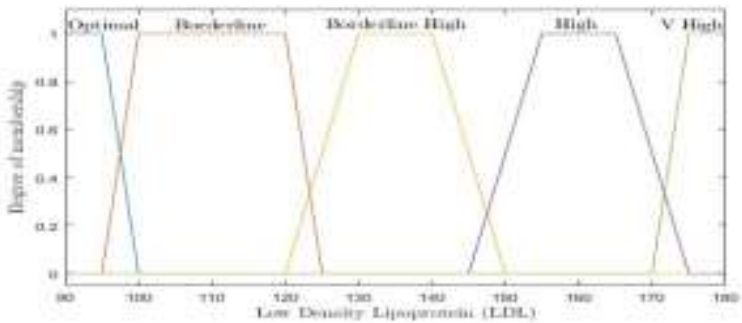

Fig. 5: Classification of LDL

\section{IV - -Body Weight:}

It refers to a person body weight; it can be modified by factors such as gender, age, degree of muscular development.

$$
\begin{aligned}
& \mu_{U w t}(x)=\left\{\begin{array}{cc}
1 & x \leq 17 \\
\frac{18.5-x}{1.5} & 17 \leq x \leq 18.5 \\
0 & x \geq 18.5
\end{array} \mu_{\text {Nwt }}(x)=\left\{\begin{array}{cc}
\frac{x-17}{3} & 17 \leq x \leq 20 \\
1 & 20 \leq x \leq 22 \\
\frac{24.9-x}{2.9} & 22 \leq x \leq 24.9 \\
0 & x \leq 17, x \geq 24.9
\end{array}\right.\right. \\
& \mu_{\text {Oweight }}(x)=\left\{\begin{array}{cc}
\frac{x-23.4}{1.6} & 23.4 \leq x \leq 25 \\
1 & 25 \leq x \leq 28 \\
\frac{29.9-x}{1.9} & 28 \leq x \leq 29.9 \\
0 & x \leq 23.4, x \geq 29.9
\end{array} \quad \mu_{\text {Obesity }}(x)=\left\{\begin{array}{cc}
0 & x \leq 28 \\
\frac{x-28}{2} & 28 \leq x \leq 30 \\
1 & x \geq 30
\end{array}\right.\right.
\end{aligned}
$$

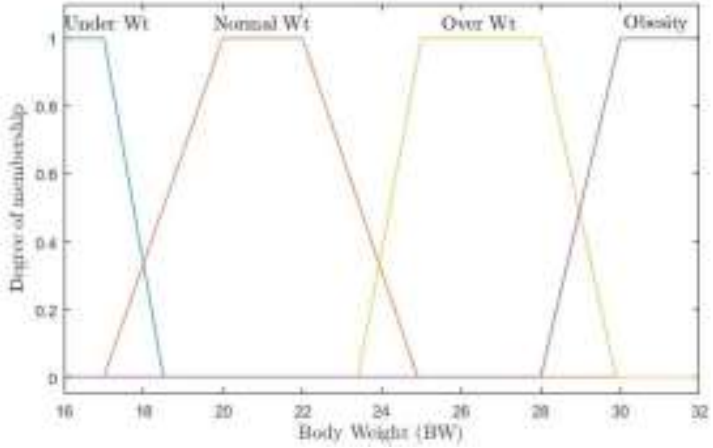

Fig. 6: Classification of Body Weight (BW)

\section{IV $_{\mathbf{5}}$-Physical Activity:}

It is defined as any bodily movement produced by skeletal a muscle that requires energy expenditure. Energy Expenditure balances the energy and weight control. It enhances the physical fitness and overall health and wellness.

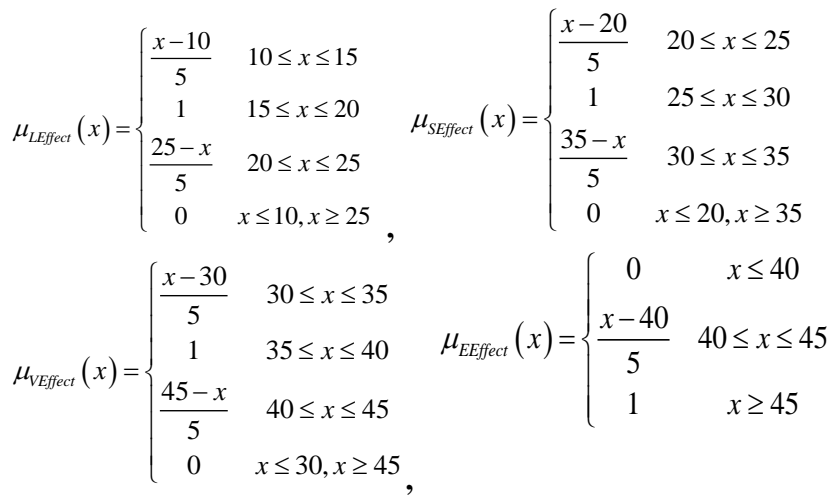

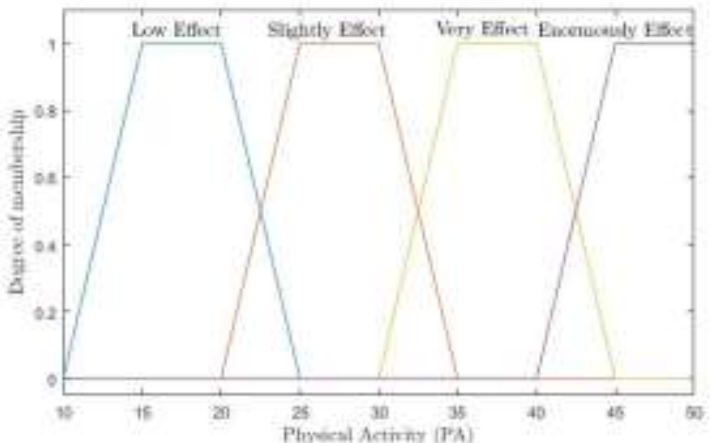

Fig. 7: Classification of Physical Activity (PA)

\subsection{Output Variables}

In this system, the given output variable Health Condition are defined and classified into linguistic variables with interval and their membership function is given by Triangular fuzzy number. Health Condition: HC is classified into seven linguistic variables with interval and triangular fuzzy number are described as follows. Consider

$$
\begin{aligned}
& \mu_{\text {VLow }}(x)=\left\{\begin{array}{cc}
\frac{x-0.1}{0.1} & 0.1 \leq x \leq 0.2 \\
\frac{0.3-x}{0.1} & 0.2 \leq x \leq 0.3 \\
0 & x \leq 0.1, x \geq 0.3
\end{array} \quad \mu_{\text {Low }}(x)=\left\{\begin{array}{cc}
\frac{x-0.2}{0.1} & 0.2 \leq x \leq 0.3 \\
\frac{0.4-x}{0.1} & 0.3 \leq x \leq 0.4 \\
0 & x \leq 0.2, x \geq 0.4
\end{array}\right.\right. \\
& \mu_{\text {Medow }}(x)=\left\{\begin{array}{cc}
\frac{x-0.3}{0.1} & 0.3 \leq x \leq 0.4 \\
\frac{0.5-x}{0.1} & 0.4 \leq x \leq 0.5 \\
0 & x \leq 0.3, x \geq 0.5
\end{array} \quad \mu_{\text {Medium }}(x)=\left\{\begin{array}{cc}
\frac{x-0.4}{0.1} & 0.4 \leq x \leq 0.5 \\
\frac{0.6-x}{0.1} & 0.5 \leq x \leq 0.6 \\
0 & x \leq 0.4, x \geq 0.6
\end{array}\right.\right. \\
& \mu_{\text {Melsevere }}(x)=\left\{\begin{array}{cc}
\frac{x-0.5}{0.1} & 0.5 \leq x \leq 0.6 \\
\frac{0.7-x}{0.1} & 0.6 \leq x \leq 0.7 \\
0 & x \leq 0.5, x \geq 0.7
\end{array} \quad \mu_{\text {Severe }}(x)=\left\{\begin{array}{cc}
\frac{x-0.6}{0.1} & 0.6 \leq x \leq 0.7 \\
\frac{0.8-x}{0.1} & 0.7 \leq x \leq 0.8 \\
0 & x \leq 0.6, x \geq 0.8
\end{array}\right.\right. \\
& \mu_{\text {versSevere }}(x)=\left\{\begin{array}{cc}
\frac{x-0.7}{0.15} & 0.7 \leq x \leq 0.85 \\
\frac{1-x}{0.15} & 0.85 \leq x \leq 1 \\
0 & x \leq 0.7, x \geq 1
\end{array}\right.
\end{aligned}
$$




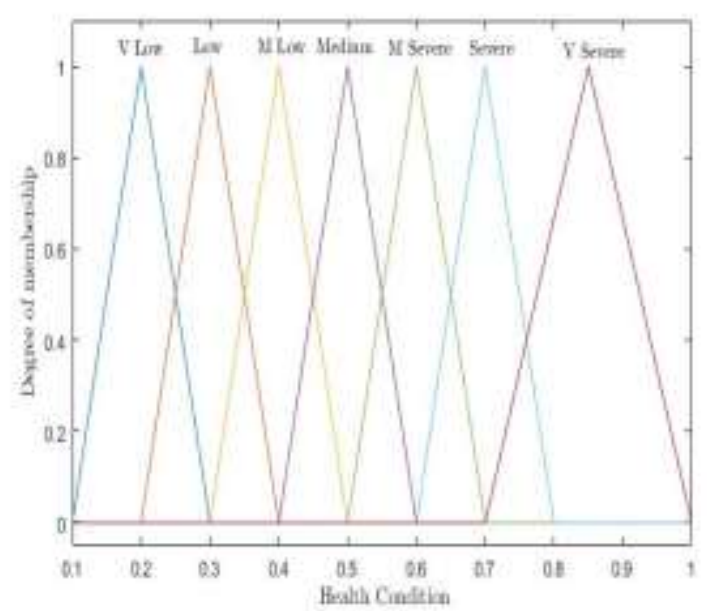

Fig. 8: Classification of Health Condition

\section{Algorithm for the Fuzzy Expert System}

Step-1

Here, $\mathrm{n}$ input factor $D_{i}, i=1,2, \ldots, n$ and $m$ outputs $R_{j}, j=1$, $2, \ldots, m$.

\section{Step-2}

Divide the input factors $D_{i}, i=1,2, \ldots, \mathrm{n}$ and $\mathrm{m}$ outputs factors $R_{j}$, $j=1,2, \ldots, \mathrm{m}$

into linguistic terms from the set $\tilde{S}=\left\{s_{i}, s_{i+1}, \ldots, s_{u}\right\}$ where

$S_{l}$ and $S_{u}$ are the lower and upper limits, $l, u=(0,1, \ldots, g)$

\section{Step-3}

Construct the suitable fuzzy membership function for each linguistic terms $D_{i}$,

$\mathrm{R}_{\mathrm{j}}$ using the available information.

\section{Step-4}

Develop $p$ If-then rules with aid of expert's $\left(E_{i}\right), i=1,2, \ldots, k$ Input- Fuzzy strings from $\mathrm{R}_{\mathrm{p}}$ and the value of the parameter $\mathrm{D}_{\mathrm{i}}$. Calculating the sting strength $w_{p}=A_{1} \wedge A_{2} \wedge \cdots \wedge A_{p}$ $\mathrm{Op}=\mathrm{p}^{\text {th }}$ rule's consequent fuzzy rules.

Output of the system $=\sum_{p=1}^{r} \frac{O_{p} w_{p}}{w_{p}}$

Step-5 Final output would be the required impact.

\section{Results and Discussion}

In type 2 diabetes, when insulin isn't available, blood glucose levels rise higher in the blood stream. Most of the people are living in India without knowing that they are affected with T2DM. Due to imprecise and vague, the diabetic factors cannot be predicted earlier. Hence, this paper deals with five factors to find the actual health condition of diabetic individual's.

Case 1: The input variables of first patient $P_{1}$ are $\{(40$, Age $),(45$, HDL), (135, LDL), (30.5, BW ), (60, PA) \}.

The linguistic string of concerned patient $\mathrm{P}_{1}$ is (Middle, Medium, Borderline High, Obesity, Enormously Effective) occurs at R608. The Rule R608 states that if Age Middle and $H D L_{\text {Medium }}$ and $L D L_{B o r-}$ derline High and $B W_{\text {Obesity }}$ and PA Enormously Effective then T2DM is Severe. Hence, the patient $P_{1}$ is affected T2DM in severe level with 0.7 membership grade.

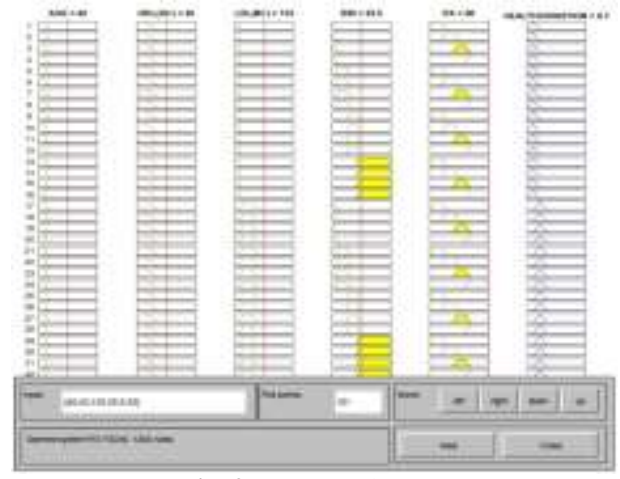

Fig. 9: Fuzzy Rule Base

\section{Conclusion}

This present study designs Fuzzy Expert System for T2DM with Linguistic term and membership functions for input variables, output variables and 1200 rule base. This system has been constructed and tested with aid of medical practitioners. The proposed Expert system would assist the medical experts for early diagnosis of the disease and proceed the treatment to save one's life.

\section{References}

[1] Ali Adeli., \& Mehdi Neshat. (2010), A Fuzzy Expert System for Heart Disease Diagnosis, Proceedings for the International MultiConference of Engineers and Computer Scientists, Vol-I, 136-139.

[2] Hasan Mir Anamul. Khaja. Sher-E-Alam., \& Ahsan Raja Chowdhury. (2010), Human disease diagnosis using a fuzzy expert system, Journal of Computing, 2(6), 66-70.

[3] Ismail Saritas., Novruz Allahverdi., \& Ibrahim Unal Sert. (2003), A Fuzzy Expert System Design for Diagnosis of Prostate Cancer, International Conference on Computer Systems and Technologies Compsystech.

[4] Kalpana. M., Senthil Kumar. A.V. (2012), Design And Implementation of Fuzzy Expert System Using Fuzzy Assessment Methodology, International Journal of Science And Applied Information Technology, 1(1), 39-45.

[5] Kandel, A. (1992), Fuzzy Expert Systems. CRC Press, Inc., Boca Raton.

[6] Maryam Mirsharif., Mahmoud Alborzi., \& Amir Ashkan Nasir Pour. (2014), IA Fuzzy Expert System \& Neuro-Fuzzy System Using Soft Computing For Gestational Diabetes Mellitus Diagnosis, Inte. J. Of Information, Security snf System Magmt., 3(1), 249-252.

[7] Najar Yousra., Ketata Raouf., \& Ksouri Mekki. (2013), A Study On The Conception Of Generic Fuzzy Expert System For Surveillance, Int. J. Of Advanced Comp. Sci. Appli., 4(8), 218-227.

[8] Nonso Nnamoko et.al. (2013), Fuzzy Expert System For Type 2 Diabetes Mellitus (T2DM) Management Using Dual Inference Mechanism, Data Driven Wellness: Association For The Advancement Of Artificial Intelligence, 67-70.

[9] Rama Devi. E., Nagaveni. N. (2010), Design Methodology of a Fuzzy Knowledgebase System to Predict the Risk Of Diabetic Nephropathy. International Journal Of Comp. Sci. Issues, 7(5), 239-247.

[10] Siler W., Buckley J. (2005), Fuzzy Expert Systems and Fuzzy Reasoning, John Wiley \& Sons, Inc., New Jersey.

[11] Tawfik Saeed Zeki., et.al, (2012), An Expert System For Diabetes Diagnosis, American Academic \& Scholarly Research Journal, 4(5) $1-13$.

[12] Zadeh, L.A. (1994), Soft Computing and Fuzzy Logic, IEEE software, $11(6), 48-56$.

[13] Zadeh, L.A., (1965), Fuzzy sets, Information and Control, 8 (1965), 338-353. 